\title{
Day-ahead Optimal Scheduling of Loads and Dispatchable Resources in a Hybrid AC/DC Microgrid of an Industrial System
}

\author{
L. Alfieri ${ }^{1}$, G. Carpinelli ${ }^{1}$, D. Proto ${ }^{1}$, G. Russo ${ }^{1}$ \\ ${ }^{1}$ Department of Electrical Engineering and of Information Technologies \\ DIETI, University of Naples "Federico II" \\ Via Claudio, 21 - 80125 - Napoli, (Italy) \\ e-mail: luisa.alfieri@unina.it, guido.carpinelli@unina.it, danproto@unina.it
}

\begin{abstract}
Industrial systems are usually characterized by high energy consumption and presence of sensitive loads which require high levels of Power Quality. Based on the grid architecture of an actual Italian industrial facility, this paper proposes the installation of a hybrid AC/DC microgrid. The hybrid microgrid includes dispatchable and non-dispatchable renewable generation units, battery storage systems and controllable loads. An optimization model is formulated to solve the problem of the day-ahead optimal scheduling of the microgrid. The model aims to optimize the operation of dispatchable resources, controllable loads and storage systems minimizing the daily costs of the energy imported from the AC grid and the costs required by the dispatchable generators while satisfying operational constraints such as those related to the production process of the industry facility. Different case studies are investigated in order to show the feasibility and effectiveness of the proposed procedure.
\end{abstract}

\section{Key words}

AC/DC microgrid, energy management, energy storage, distributed generation, day-ahead scheduling.

\section{Introduction}

In the last decade significant modifications to the structure and management of power systems have been experimented and we can expect that the future electrical distribution systems progressively approach the new concepts of Smart Grids (SGs) and Microgrids ( $\mu \mathrm{Gs}$ ). These modifications are mainly due to the increasing level of penetration of dispersed generation, storage systems and controllable loads together with the use of Information and Communication Technologies [1-6].

As well known, $\mu$ Gs can be classified in AC and DC $\mu \mathrm{Gs}$ depending on the characteristics of the supply voltage [2-4]. AC $\mu \mathrm{Gs}$ have the great benefit of utilizing existing AC grid technologies. However, DC $\mu$ Gs seem particularly suitable for supporting modern needs, because most distributed generation sources can generate DC power directly. Moreover, DC grids can provide high-continuity and high-quality power levels to AC sensitive loads [4-7]. Recently, hybrid DC/AC $\mu$ Gs have been considered; they include both DC and AC sections and consequently take advantages of both them [8-11].
Hybrid DC/AC $\mu \mathrm{Gs}$ can be useful also in industrial systems where usually only AC grid are installed. However, the introduction of DC sections seems particularly suitable due to the above mentioned evidences. Then, this paper proposes the implementation of a hybrid AC/DC $\mu \mathrm{G}$ including both $\mathrm{DC}$ and $\mathrm{AC}$ sections in an actual Italian industrial facility. The hybrid $\mu \mathrm{G}$ includes dispatchable and renewable generation units, a storage system and controllable loads.

The hybrid AC/DC $\mu \mathrm{G}$ requires to adopt suitable strategies to optimally control the contemporaneous presence of different technologies such as AC and DC energy generation systems, storage systems and controllable loads. These optimization strategies are aimed at operating the grid efficiently and should take into account technical and operating limitations of both the grid and its resources.

The solution of linear and/or non-linear optimization models has been usually required to apply the optimal strategies whose formulation can be characterized by different time horizons. In the technical literature, dayahead scheduling or real-time procedures were proposed separately [12-15]. In particular, the first type of procedure operates on a day-ahead basis and aims to schedule controllable resources to preserve reliability during operation. The real-time procedure aims to guarantee balance between load and production on a very short time basis.

Suitable control strategies were proposed in the technical literature also for hybrid AC/DC $\mu$ Gs. In [8] a cooperative control is proposed to enhance power quality, where the AC and DC $\mu \mathrm{Gs}$ are treated as two separate entities. In [9], an algorithm for the coordination and control of the $\mu \mathrm{G}$ is applied aimed at minimizing the power transfer between the AC and DC grids, and guaranteeing the correct operation of both sub-grids. In [10], an optimal day ahead scheduling is proposed with the aim of minimizing the total cost sustained during the day. In [11], a real time control of the active and reactive power supplied by the interlinking AC/DC converter is presented, aimed at proportionally sharing the power among the different power sources on the basis of their ratings. 
This paper proposes a new day-ahead scheduling strategy to optimally control the proposed hybrid AC/DC $\mu \mathrm{G}$. The day-ahead scheduling is performed using a centralized control system (CCS) and aims to optimally operate the controllable loads, dispatchable generation units, and battery energy storage systems included in the proposed hybrid $\mu \mathrm{G}$. An adequate optimization problem is formulated and solved, taking into account technical and operational constraints as well as guaranteeing the minimization of the costs sustained by the industrial facility for the total daily energy required.

The main novelty of the proposal of this paper is that, unlike the control algorithms discussed in [8-11], the proposed approach allows handling controllable loads and dispatchable generations. Moreover, compared to the proposals of [8] and [11], the proposed control operates on the AC and DC sub grids contemporaneously thus considering the hybrid $\mu \mathrm{G}$ as a whole with all its complex devices.

The paper is organized as follows: Section II describes the hybrid AC/DC $\mu \mathrm{G}$ considered for the study while Section III details the day-ahead scheduling optimization model and the solution procedure. Section IV shows the first results of a practical application of the proposed procedure.

\section{The hybrid microgrid under study}

A general scheme of the proposed AC/DC hybrid $\mu \mathrm{G}$ is shown in Fig. 1. It includes linear and non-linear loads, dispatchable and non-dispatchable dispersed generation units and energy storage systems. A DC/AC static converter is used to connect the DC grid with the AC grid. Non-dispatchable DC generators, sensitive loads and BESSs are connected to the DC grid.

Sensitive loads connected to the DC grid are also controllable loads. It is assumed that they have an assigned (known) pattern of power consumption, i.e., once the device is turned on (start-up time), it works according to its own pattern of power consumption until the job is completed. In the formulation proposed in this paper, the patterns of load power are calculated from the known rated powers, or are predicted based on historical data of the loads; look-up tables also can be used to determine these values. The start-up times of the loads are control variables in the optimization problem (i.e., the loads are controllable in terms of start-up time).

Dispatchable AC generators and linear/non-linear AC loads are connected to the AC grid.

In particular:

i) sensitive loads are connected to the $\mathrm{DC} \mu \mathrm{G}$ through $\mathrm{DC} / \mathrm{AC}$ converters;

ii) the DC generators are non-dispatchable photovoltaic systems connected to the DC grid through DC/DC converters and equipped with a Maximum Power Point Tracker (MPPT);

iii) the storage system is a battery connected to the DC grid through a DC/DC converter and can be controlled in terms of charging/discharging power;

iv) the $\mathrm{AC}$ generators, which are dispatchable generation units, are equipped with microturbines connected to the AC grid through AC/AC static converters.
The CCS in Fig. 1 performs the day-ahead control strategy for the optimal operation of controllable loads, dispatchable generation units, and battery energy storage systems (BESSs). The day-ahead scheduling minimizes the total daily costs of energy including the cost of the imported electrical energy and the cost of production of the dispatchable generation while satisfying operational constraints as those related to the production process.

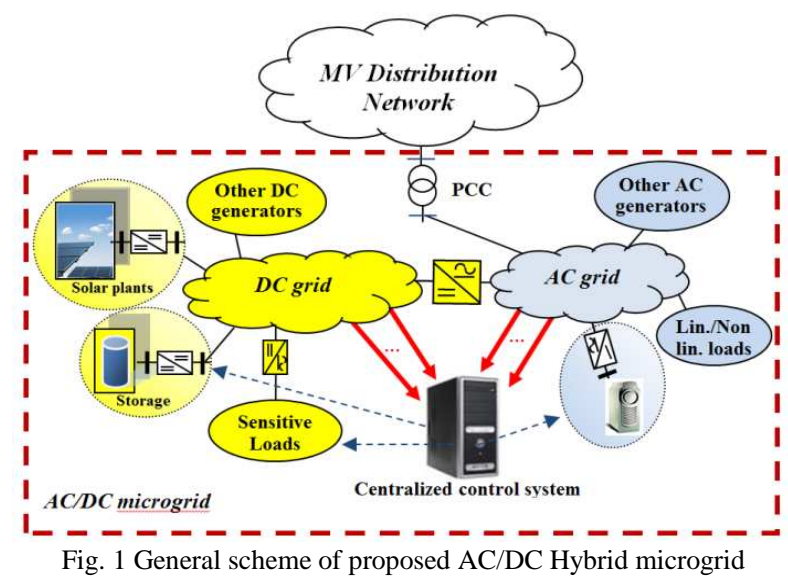

\section{The day-ahead optimization problem formulation}

The optimal scheduling of the considered hybrid $\mu \mathrm{G}$ is formulated by means of a non-linear optimization problem involving both integer and continuous variables. In the formulation described below it is supposed that $K$ BESSs, $R$ non-dispatchable generators and $M$ controllable loads are connected to the DC $\mu \mathrm{G}$, while $N$ dispatchable generators and $P$ non-controllable loads are connected to the AC $\mu \mathrm{G}$, The optimization time interval is one day (24 hours) that is discretized into $n_{t}$ uniform time slots (e.g., $n_{t}=24 \times 3$ if 20 minutes per slot are considered).

Inputs of the optimization problem are the day-ahead forecasted power of non-dispatchable units and noncontrollable loads at each time interval of the day, the BESS state of charge at the beginning of the day and the data regarding the controllable loads that are: the required number of work cycles in a day $\left(N_{\text {cycle }}\right)$, the duration of each work cycle in terms of number of time slots $\left(N_{\text {slot }}\right)$ and the absorbed energy profile during each cycle. To better clarify the meaning of these data, Fig. 2 presents an example of the daily controllable load power profile with the load in service three times in a day $\left(N_{\text {cycle }}=3\right)$ and each load profile lasting two time slots $\left(N_{\text {slot }}=2\right)$.

Further inputs refer to the parameters of the cost function used for the dispatchable generators and the hourly price of the electricity related to the tariff scheme adopted by the facility (e.g., real time or time of use pricing). 


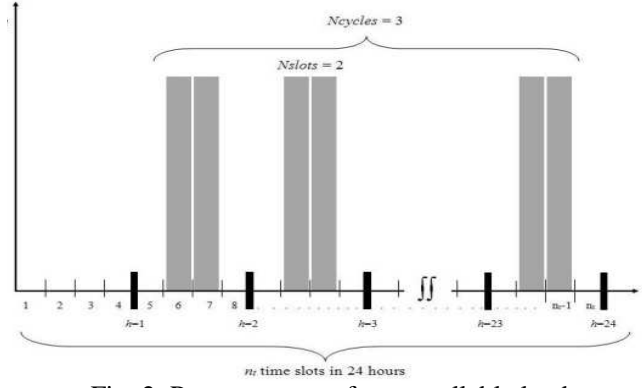

Fig. 2. Power pattern of a controllable load

Outputs of the procedure are the daily power profile of the dispatchable generator units, the charge/discharge power profile of the BESS along the day and the power profile of the controllable loads.

The day-ahead scheduling is formulated in terms of single objective optimization aimed at minimizing an objective function while satisfying equality and inequality constraints. The objective function to be minimized is the cost sustained by the industrial facility for the total daily energy which includes the cost for the energy imported from the MV grid and the production cost of the dispatchable generation. Then, the objective function can be formulated as:

$f_{o b j}(\mathbf{x})=\Delta t \sum_{j=1}^{n_{t}} \operatorname{Pr}_{E n, j} \cdot P_{j}^{g r i d}+\Delta t \sum_{j=1}^{n_{t}} \sum_{n=1}^{N} C_{n, j}^{d G}$

where $P_{j}^{\text {grid }}$ is the grid active power absorbed at the $j^{\text {th }}$ time slot, $\operatorname{Pr}_{E n, j}$ is the day ahead forecasted electricity price and $C_{n, j}^{d G}$ is the production cost function of the $n^{\text {th }}$ dispatchable generator at the $j^{\text {th }}$ time slot. Usually, the production cost related to the generation of the dispatchable generator is evaluated by means of a quadratic function [16], thus in the considered application it is assumed that:

$C_{n, j}^{d G}=a_{A C G, n} P_{A C G, n, j}{ }^{2}+b_{A C G, n} P_{A C G, n, j}+c_{A C G, n} u(n, j)$

where $a_{A C G, n}, b_{A C G, n}$ and $c_{A C G, n}$ are specified parameters of the $n^{\text {th }}$ generator, $P_{A C G, n, j}$ is the power supplied to the grid by the dispatchable generator and $u(n, j)$ refers to a binary discrete variable which denotes whether the $n^{\text {th }}$ dispatchable AC generation unit is ON or OFF at time slot $j$. The equality and inequality constraints are shown for the DC and AC $\mu$ Gs separately.

\section{$D C \mu G$ constraints}

The energy stored in the $k^{\text {th }} \operatorname{BESS}(k=1,2, \ldots, K)$ has to be the same at the beginning and end of the day:

$\sum_{j=1}^{n_{t}} \gamma_{l, k} P_{B E S S, j, k} \Delta t=0$

$$
\gamma_{l, k}= \begin{cases}\frac{1}{\eta_{\text {bess }, k}} & \text { if } P_{B E S S, l, k} \leq 0 \\ \eta_{\text {bess }, k} & \text { if } P_{B E S S, l, k}>0\end{cases}
$$

where $P_{B E S S, j, k}$ is the power of the $k^{\text {th }}$ BESS at the time slot $j$ and $\eta_{\text {bess, } k}$ is the efficiency of the $k^{\text {th }}$ BESS. In each time slot, the power charged/discharged by the BESS is bounded by the battery size:

$$
\begin{array}{cc}
-P_{\max }^{d c h} \leq P_{B E S S, j, k} \leq 0 & j \in \Omega_{d c h, k} \\
0 \leq P_{B E S S, j, k} \leq P_{\max }^{c h} & j \in \Omega_{c h, k}
\end{array}
$$

where $\Omega_{d c h, k}\left(\Omega_{c h, k}\right)$ is the set of time slots in which the battery is allowed to charge (discharge), $P_{\max }^{c h}$ and $P_{\max }^{d c h}$ are the maximum power rates in charging and discharging stages, respectively. It has to be noted that, for each battery, the choice of $\Omega_{d c h, k}$ and $\Omega_{c h, k}$ is related to the admissible number of charging/discharging cycles per day. As an example, in case of one cycle/day, the battery can be charged during the early morning and night (i.e., low price hours) and discharged during the central hours of the day (i.e., high price hours).

For the $k^{\text {th }}$ BESS, further constraints are imposed to the energy stored in the battery that, at each time slot $j$, has to be within an admissible range $\left[S O C_{s p, k}^{\min }, S O C_{s p, k}^{\max }\right]$ :

$S O C_{s p, k}^{\min } \leq S O C_{s p, k}^{\mathrm{in}}+\sum_{l=1}^{j} \gamma_{l, k} P_{B E S S, l, k} \Delta t \leq S O C_{s p, k}^{\max }$

where $S O C_{s p, k}^{\text {in }}$ is the energy stored in the $k^{\text {th }}$ BESS at the beginning of the day. The value of $S O C_{s p, k}^{\max }$ is due to the battery size, and the value of $S O C_{s p}^{\min }$ can be properly selected in order to take into account the maximum allowable depth of discharge, thus accounting also for the preservetion of the battery lifetime [17].

In an industrial facility, as it is the case of the application considered in this paper, the controllable loads can be in service only in specified sets of time slots, which are typically linked to the specified daily work cycle. Hence, the power absorbed by the $m^{\text {th }}(m=1,2$, ...M) controllable load at the $j^{\text {th }}\left(j=1,2, \ldots n_{t}\right)$ time slot is constrained by:

$0 \leq P_{\text {CLoad }, j, m}^{D C} \leq P_{\text {CLoad }, m}^{D C m a x} j \in \Omega_{W H, m}$

where $\Omega_{W H, m}$ is the set of time slots of the planned daily work hours and $P_{C L o a d, j, m}^{D C}$ is the power absorbed by the $m^{\text {th }}$ controllable load at the $j^{\text {th }}$ time slot. The following constraints are imposed during the rest of the day:

$P_{C L o a d, j, m}^{D C}=0, \quad m=1,2, \ldots M j \notin \Omega_{W H, m}$

The power absorbed by the $m^{\text {th }}$ controllable load has to satisfy the following equations:

$\sum_{j=1}^{n_{t}} P_{C L o a d, j, m}^{D C} \Delta t=E_{\text {Load }, m}^{d}$

$\sum_{j=l_{m}}^{l_{m}+N_{\text {slot }, m^{-1}}} P_{\text {CLoad }, j, m}^{D C} \Delta t=E_{\text {Load }, m}^{d} / N_{\text {cycle }, m}$ $l_{m} \in \Omega_{m}$

where, with reference to the $m^{\text {th }}$ controllable load, $l_{m}$ is the time slot associated with the start up time of the controllable load, $N_{\text {cycle, } m}$ is the required number of work cycles in a day, $N_{\text {slot }, m}$ is the duration of each work cycle in terms of number of time slots, $E_{\text {Load, } m}^{d}$ is the total daily energy request, and $\Omega_{m}$ is the set of $N_{\text {cycle, } m}$ time slots associated with start up times of the controllable load.

The power balance at the DC side of the $\mu \mathrm{G}$ at the $j^{\text {th }}$ time slot is:

$P_{A C-D C, j}=$

$\sum_{k=1}^{K} P_{B E S S, j, k}-\sum_{r=1}^{R} P_{D C G, j, r}+\sum_{m=1}^{M} P_{C L o a d, j, m}^{D C}$

(9) 
where $P_{D C G, j, r}$ is the power injected in the DC $\mu \mathrm{G}$ by the $r^{\text {th }}$ generator at the $j^{\text {th }}$ time slot and $P_{A C-D C, j}$ is the power flowing, at each time slot $j$, through the AC-DC interconnection converter, that is positive when flowing from $\mathrm{AC}$ to $\mathrm{DC} \mu \mathrm{G}$, and whose value is limited by the converter size $\left(P_{\text {size }}^{\text {conv }}\right)$ :

$\left|P_{A C-D C, j}\right| \leq P_{\text {size }}^{\text {conv }}$

\section{$A C \mu G$ constraints}

At each time slot $j$, the power supplied by the $n^{\text {th }}$ dispatchable generator has to fall into an admissible range $\left[P_{\min }^{d G}, P_{\max }^{d G}\right]$ :

$u_{j, n} P_{\min , n}^{d G} \leq P_{A C G, j, n} \leq u_{j, n} P_{\max , n}^{d G}$

where the upper value is related to the size of the dispatchable generator, while the lower limit is due to operational constraints and the type of generator [16].

The power balance at the $\mathrm{AC}$ side of the $\mu \mathrm{G}$ at the $j^{\text {th }}$ time slot is:

$P_{j}^{g r i d}=\sum_{p=1}^{P} P_{N C L o a d, j, p}^{A C}+P_{A C-D C, j}-\sum_{r=1}^{R} P_{A C G, j, r}$

where $P_{N C L o a d, j, p}^{A C}$ is the power absorbed by the $p^{\text {th }}$ AC noncontrollable load and $P_{A C G, j, r}$ is the power supplied to the AC $\mu \mathrm{G}$ by the $r^{\text {th }}$ dispatchable generator. A constraint is also imposed on the maximum values of the power that can be imported from/exported $\left(P_{M A X}^{\text {grid }_{\text {in }}}\right.$ and $P_{M A X}^{\text {grid }}$ out , respectively) to the distribution grid as:

$-P_{M A X}^{\text {grid }_{\text {out }}} \leq P_{j}^{\text {grid }} \leq P_{M A X}^{\text {grid }_{\text {in }}}$

where the bounds can be related to the size of the AC network interfacing transformer, contractual agreements or peak shaving services.

Note also that, in case the hybrid $\mu \mathrm{G}$ is allowed to export power to the distribution grid, the (1) could be slightly modified since, in the present form, it involves the same price for both bought and sold powers. It has to be underlined also that, in the proposed day-ahead procedure the DC and AC grid internal constraints are neglected since they require a cumbersome formulation of the network equations, state variables, and constraints.

This simplification does not introduce a significant error because industrial systems usually are characterized by reduced-size grids, so the line impedances do not influence the amplitude of the bus voltage significantly.

\section{Numerical results}

The scheduling strategy illustrated in Section 3 was applied to the AC/DC Hybrid microgrid $(\mu \mathrm{G})$ shown in Fig. 3.

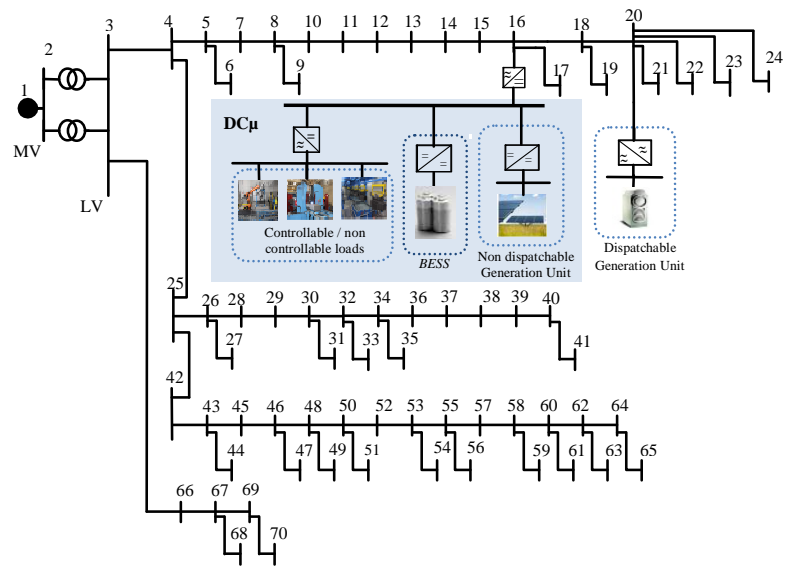

Fig. 3 General scheme of industrial AC/DC $\mu \mathrm{G}$

This is the electrical distribution system of the GETRA, an industrial facility located in the South Italy. Its electrical distribution system includes four, lowvoltage electrical lines ("tanks and boxes manufactory", "tests", "assembly" and "winding and coils" lines) fed by two MV/LV transformers. Each electrical line is dedicated to a different manufacturing process.

In particular, the AC/DC $\mu \mathrm{G}$ included three controllable loads, a $60-\mathrm{kWp}$ PV generation plant, a 30 $\mathrm{kVA}$ micro turbine generator and a $100-\mathrm{kWh}$ storage system (BESS). An AC/DC, PWM-controlled, static converter was used to connect the DC and AC $\mu \mathrm{Gs}$.

The day was divided into 20-min time slots (i.e. the number of daily slots is equal to 72). The controllable loads are AC loads, and they are connected to the DC $\mu \mathrm{G}$ through a dedicated line and a DC/AC converter that supplies them. The controllable loads (whose characteristics are reported in Table I) are:

- automated bending robot for metal plates (ABRMP)

- sandblasting machine (SM)

- PLCs used to control the automation of all of the electromechanical processes.

Among the commercially-available batteries, the Li-ion battery was chosen and installed. This choice was based on the specific characteristics of this type of battery, i.e., a long lifetime, even for a significant depth of discharge, and its high efficiency. The storage system limits are $S O C_{s p, k}^{\min }=20 \mathrm{kWh}, S O C_{s p, k}^{\max }=100 \mathrm{kWh}$ with $P_{\text {max }}=20$ $\mathrm{kW}$; the BESS charging and discharging efficiencies are equal to 0.92 . To guarantee an adequate state of charge at the beginning of the work hours, $S O C_{s p}^{\text {in }}$ was fixed equal to $100 \mathrm{kWh}$. In addition, the time interval for BESS discharging was fixed from 6:00 A.M. to 10:00 P.M. and that for BESS charging from 10:00 P.M. to 6:00 A.M. The micro turbine power limits were imposed to be $P_{\text {min }}^{d G}=0$ and $P_{\max }^{d G}=30 \mathrm{~kW}$.

TABLE I

CHARACTERISTICS OF THE LOADS OF THE DC MICRO GRID

\begin{tabular}{c|c|c|c|c|c}
\hline \hline Load & $\begin{array}{c}P \\
{[\mathrm{~kW}]}\end{array}$ & $N_{\text {cycle }}$ & $N_{\text {slot }}$ & $\begin{array}{c}\text { Cycle } \\
\text { duration }\end{array}$ & Working hours \\
\hline ABRMP & 20 & 1 & 12 & $1 \mathrm{~h}$ & $\begin{array}{c}\text { 6:00 A.M.to } \\
\text { 10:00 P.M. }\end{array}$ \\
\hline SM & 50 & 5 & 3 & $20 \mathrm{~min}$ & $24 \mathrm{~h}$ \\
\hline PLCs & 2 & 1 & 72 & $20 \mathrm{~min}$ &
\end{tabular}

Several operating conditions were analyzed; for the sake of conciseness, only some results of the case that 
refers to the real time energy price profile shown in Fig. 4 are reported. Fig. 5 shows the input data of the optimization model, with reference to the forecasted values of the powers of non-controllable loads and the PV unit.

The output data of the applied scheduling strategy are those shown in Figs. from 6 to 9; they refer to the active powers of the microturbine (Fig. 6), storage system (Fig. 7), controllable loads (Fig. 8) and that flowing through the AC-DC converter (Fig. 9). Figs. from 6 to 9 clearly show that:

- Controllable DC loads are turned on in the time slots where the energy price is lower, i.e. at 6 A.M. up to 8 A.M. and at 6 P.M. up to 10 P.M.;

- in the hours in which the energy price is higher (from 10 A.M. to 6 P.M.), the $\mu G^{\prime}$ 's resources (i.e., microturbine, PV and storage systems) are used to supply power to the loads;

- in the time 8 A.M. to 6 P.M. the DC $\mu \mathrm{G}$ export energy to the $\mathrm{AC} \mu \mathrm{G}$.

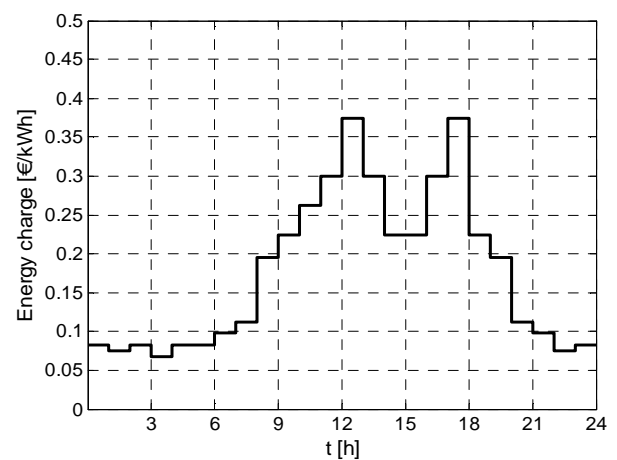

Fig. 4 Energy charge profile

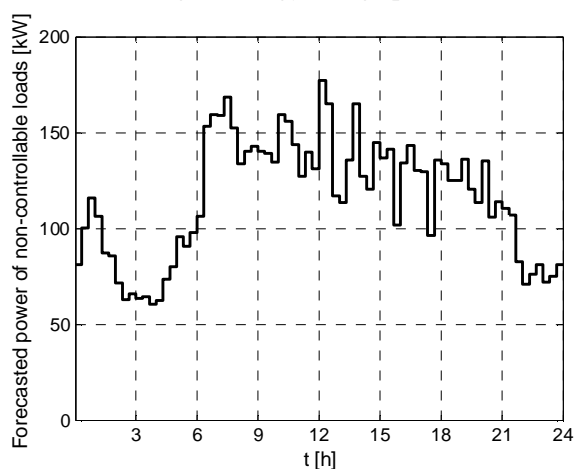

(a)

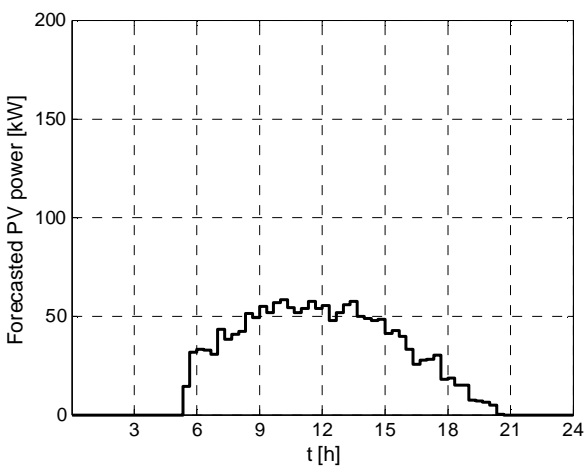

(b)

Fig. 5. Day-ahead predicted non-controllable load power (a) and PV power (b)

Fig. 10 shows the hourly cash flows of the industrial facility without (solid line) and with (dashed lines) the proposed hybrid $\mu \mathrm{G}$. Fig. 10 clearly evidences the reduction of energy costs from 7 A.M. to 7 P.M. thanks to the reduction of the power imported from distribution network in high energy price hours. Globally, the total daily costs sustained by the industrial facility without the support of the $\mu \mathrm{G}$ are $29 \%$ greater than those sustained with the proposed strategy.

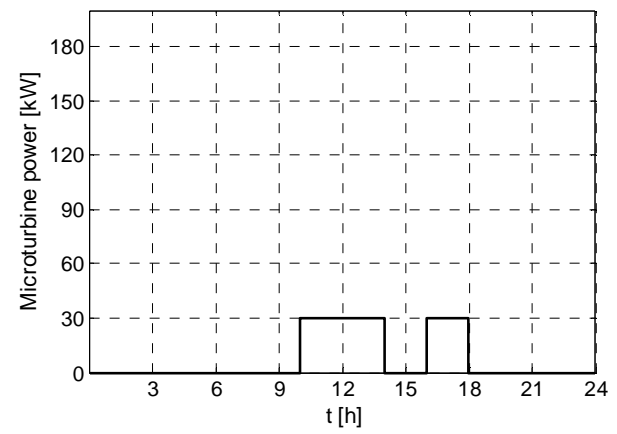

Fig. 6. Scheduled powers of Microturbine

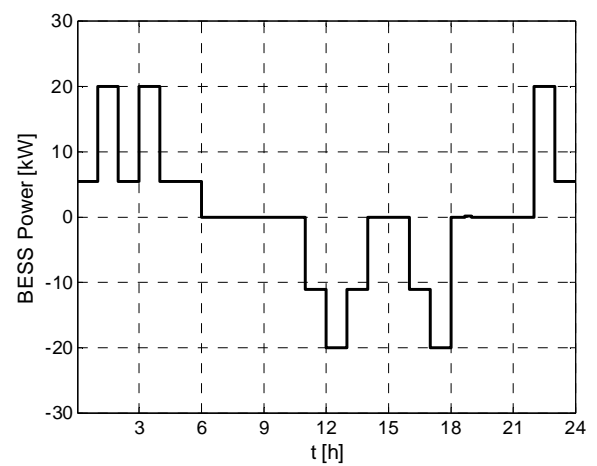

Fig. 7. Scheduled powers of BESS

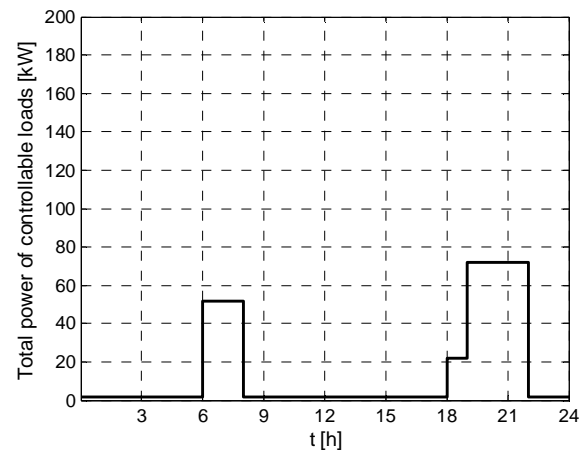

Fig. 8. Scheduled powers of total controllable loads

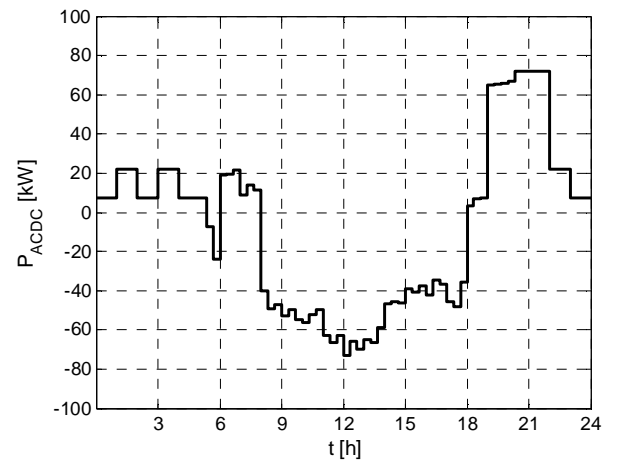

Fig. 9. Scheduled power profile at the AC-DC interconnection point 


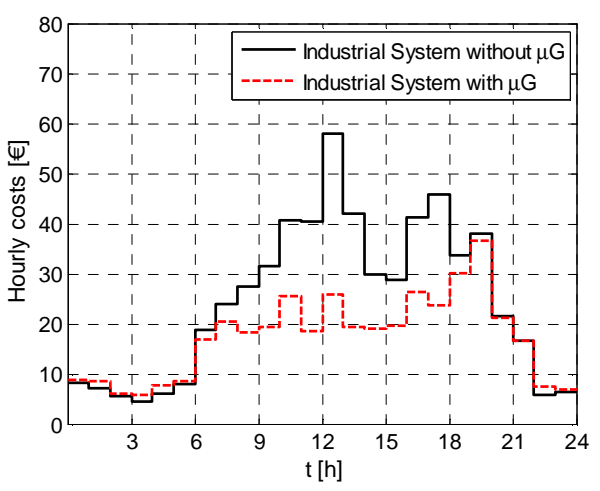

Fig. 10. Cash flow of industrial system without (solid line) and with (dashed lines) $\mu \mathrm{G}$

\section{Conclusions}

This paper proposes a modification of an actual industrial system with the installation of a hybrid AC/DC $\mu \mathrm{G}$ including dispatchable generation units, PV generation systems, storage systems and controllable loads.

An optimization model is formulated to solve the problem of the day-ahead optimal scheduling. The model aims to optimize the operation of dispatchable resources and controllable loads minimizing the daily costs of the energy imported from the AC grid as well as those required by the dispatchable generation and satisfying operational constraints such as those related to production process requirements.

The main outcomes of the paper are that:

- the proposed control scheme allows optimally managing different resources and controllable loads furnishing also high continuity to sensible loads;

- the hybrid $\mu \mathrm{G}$ in an industrial facility applying the proposed control strategy makes it possible to obtain significant economic benefits.

\section{Acknowledgement}

This work was supported by MIUR (Ministero dell'Istruzione dell'Università e della Ricerca) in the frame of PROGETTO PON R\&C DD 713/Ric. del 29/10/2010 Titolo III - Creazione di nuovi Distretti e/o nuove Aggregazioni Pubblico-Private. Progetto di ricerca industriale, sviluppo sperimentale e formazione PON03PE_00178_1: Microgrid Ibride in Corrente Contiuna e Corrente Alternata (MICCA).

\section{References}

[1] E. Santacana, G. Rackliffe, Xiaoming Feng, "Getting Smart", IEEE Power and Energy Magazine, Vol. 8, No. 2, pp. 41-48, 2010

[2] H. Farhangi, "The Path of the Smart Grid", IEEE Power and Energy Magazine, Vol. 8, No. 1, pp. 18-28, 2010

[3] N. Hatziargyriou, H. Asano, R. Iravani, C. Marnay, "Microgrids", IEEE Power and Energy Magazine, Vol.5, No.4, pp.78-94, 2007
[4] K. Kurohane, T. Senjyu, A. Yona, N. Urasaki, T. Goya, T. Funabashi: "A Hybrid Smart AC/DC Power System", IEEE Transactions on Smart Grid, Vol. 1, No.2, pp.199204, Sept. 2010

[5] P. Basak, S. Chowdhury, S. H. Dey, S.P. Chowdhury: "A literature review on integration of distributed energy resources in the perspective of control, protection and stability of microgrid", Renewable and Sustainable Energy Reviews, Vol. 16, no. 8, October 2012, Pages 5545-5556

[6] E. Planas, A. Gil-de-Muro, J. Andreu, I. Kortabarria, I. M. de Alegría: "General aspects, hierarchical controls and droop methods in $\mu$ Gs: A review", Renewable and Sustainable Energy Reviews, Vol. 17, January 2013, Pages 147-159

[7] G. Carpinelli, A. Bracale, P. Caramia, "The GREAT project: Integer linear programming-based day-ahead optimal scheduling of a DC microgrid", 12th International Conference on Environment and Electrical Engineering (EEEIC), pp.573,578, 5-8 May 2013.

[8] J.M. Guerrero, Poh Chiang Loh, Tzung-Lin Lee, M. Chandorkar, "Advanced Control Architectures for Intelligent Microgrids-Part II: Power Quality, Energy Storage, and AC/DC Microgrids," Industrial Electronics, IEEE Transactions on, vol.60, no.4, pp.1263,1270, April 2013

[9] Xiong Liu, Peng Wang, Poh Chiang Loh, "A Hybrid AC/DC Microgrid and Its Coordination Control," Smart Grid, IEEE Transactions on, vol.2, no.2, pp.278,286, June 2011

[10] P.T. Baboli, M. Shahparasti, M.P. Moghaddam, M.R. Haghifam, M. Mohamadian, "Energy management and operation modeling of hybrid AC-DC microgrid," IET Generation, Transmission \& Distribution, vol. 8, no. 10, pp. 1700-1711, 2014

[11] P.C. Loh, Ding Li, Yi Kang Chai, F. Blaabjerg, "Autonomous Operation of Hybrid Microgrid With AC and DC Subgrids," Power Electronics, IEEE Transactions on, vol.28, no.5, pp.2214,2223, May 2013

[12] Hongyu Wu, M. Shahidehpour, M.E. Khodayar, "Hourly Demand Response in Day-Ahead Scheduling Considering Generating Unit Ramping Cost," Power Systems, IEEE Transactions on, vol.28, no.3, pp.2446,2454, Aug. 2013

[13] M. Doostizadeh and H. Ghasem, "Day-ahead scheduling of an active distribution network considering energy and reserve markets," Euro. Trans. Electr. Power; vol. 23 , pages 930-945, 2013

[14] S. A. Pourmousavi, M.H. Nehrir, "Real-Time Central Demand Response for Primary Frequency Regulation in Microgrids, " Smart Grid, IEEE Transactions on, vol.3, no.4, pp.1988,1996, Dec. 2012

[15] C.M. Colson, M.H. Nehrir, M.H., "A review of challenges to real-time power management of microgrids," Power \& Energy Society General Meeting, July 2009

[16] Binyan Zhao, Yi Shi, Xiaodai Dong, Wenpeng Luan, J. Bornemann, "Short-Term Operation Scheduling in Renewable-Powered Microgrids: A Duality-Based Approach," Sustainable Energy, IEEE Transactions on , vol.5, no.1, pp.209,217, January 2014

[17] K.C. Divya, J. Østergaard, "Battery energy storage technology for power systems: An overview," Electric Power System Research, Vol. 79, pp. 511-520, 2009. 